\title{
Evaluating the Reliability of Assessing Home-Packed Food Items Using Digital Photographs and Dietary Log Sheets
}

\author{
Alain P. Gauthier, BEPS, PhD; Bridget T. Jaunzarins, BPHE; \\ Sarah-Jane MacDougall, BEd; Michelle Laurence, MHK; J. Lynn Kabaroff, MHK; \\ Alison A. Godwin, PhD; Sandra C. Dorman, PhD
}

\begin{abstract}
Objective: To assess the reliability of manual data entry for home-packed food items by using digital photographs and dietary log sheets.

Methods: Data from 60 lunches were entered by researcher A and B independently. Researcher B re-entered researcher A's items within 1 week. Researcher B then re-entered her items 4 weeks from the initial entry point.

Results: The inter-rater reliability intraclass correlation coefficient (ICC) was 0.83 for total kilocalories and ranged from $0.75-0.87$ for macronutrients. The intra-rater reliability ICC was 0.92 for total kcal and ranged from $0.90-0.92$ for macronutrients. The inter-rater ICCs for the 5 selected micronutrients ranged from 0.33-0.83, whereas the intra-rater ICCs for these micronutrients ranged from 0.65-0.98.

Conclusions and Implications: This method of data entry is feasible and its reliability is promising for macronutrient investigations. Continued assessment of this method for investigations related to micronutrient content is recommended.
\end{abstract}

Key Words: children, nutrition software, lunch, photographs, reliability (J Nutr Educ Behav. 2013; 45:708-712.)

\section{INTRODUCTION}

Collecting accurate and reliable data about food consumption can disrupt eating behaviors and presents several challenges to researchers. Traditional methods of assessing food intake have included dietary records, 24-hour dietary recall, food frequency questionnaires, and diet history, all of which have been found to be limited by their reproducibility and are generally accepted to underreport or overreport actual eating habits. ${ }^{1,2}$ The challenges of reproducibility are related to the fact that people experience difficulty accurately recalling food consumed, cannot estimate portion size, and have trouble reporting the frequency of food consumption. ${ }^{3,4}$ Furthermore, data entry errors may occur, and in the case of children, many studies rely on parents as proxy reporters, ${ }^{1,3}$ further leading to error in recording data.

More recently, researchers have turned to technological advances, 5,6 including the use of digital photography, to assess food behavior. ${ }^{7-10}$ Digital photographs have shown promise as an efficient and noninvasive method of data collection. ${ }^{5}$ Using photographs, however, relies in part on the researchers' ability to properly interpret the content of the photograph and in some cases accurately transfer the information from the photograph to a nutrition analysis program. Martin et $\mathrm{al}^{7}$ used digital photographs and a US Department of Agriculture computer application program to assess food intake among 43 sixth-graders in a cafeteria setting, finding high inter-rater agreement between 2 registered dietitians for kilocalorie identification (intraclass

School of Human Kinetics, Laurentian University, Sudbury, Ontario, Canada

Address for correspondence: Alain P. Gauthier, BEPS, PhD, Laurentian University, School of Human Kinetics, 935 Ramsey Lake Rd, Sudbury, Ontario, Canada P3E 2C6; Phone: (705) 675-1151, extension 1071; Fax: (705) 675-4845; E-mail: agauthier@laurentian.ca (C)2013 SOCIETY FOR NUTRITION EDUCATION AND BEHAVIOR http://dx.doi.org/10.1016/j.jneb.2013.05.005

correlation $=0.95$ ). In a similar context, Swanson ${ }^{8}$ assessed 5,394 items from meal images taken in a cafeteria setting. Two independent raters were used to compare estimates of how much of each item was consumed. The 2 raters identified the quantity consumed to the nearest $10 \%$ increment. For $99 \%$ of the items $(\mathrm{n}=$ 5,340 ), the raters were within three $10 \%$ increments of each another. An important limitation of these studies is that they relied on a "controlled" cafeteria-style environment. In Canada, elementary schools rely primarily on home-packed lunches. Assessing self-prepared foods comes with a series of additional challenges, including high individual variability in food content, limited consistency in portion size, and some uncertainty regarding the origin of items, especially in the absence of food labels.

Given that obesity trends among children have reached epidemic proportions $^{11,12}$ and that children spend approximately one half of their waking hours in school, assessing school nutrition in isolation is of great importance. Thus, the purpose of the current study was to assess the reliability of manual data entry by 
different evaluators for home-packed food items over time by using digital photographs and dietary log sheets.

\section{METHODS}

\section{Participants and Data Source}

Students in grades 3-6 from 2 neighboring schools were invited to participate in this study. The contents of student lunches were recorded on 4 separate days by using student logs and digital photographs. First, students listed their lunch content on daily log sheets, which were individually verified by a researcher to clarify items because some students did not have strong writing skills. Students were told to include as much detail as possible, including an item count or caloric content where possible. At the end of each day, participants indicated, next to all items on their log sheet, if they "ate it all," "ate half," or "did not eat it." Second, a minimum of 2 photographs were taken of each participant's lunch before consumption, 1 showing the identifying labels of prepacked food (or food content) and the second capturing nutritional information labels on the same foods. A numbered card was included in the picture to identify the participant, indicate the study day, and to gauge portion size.

Research ethics board approval was obtained from the Laurentian University Research Ethics Board and participating school boards. Parent/ guardian consent and student assent were also obtained before collecting data.

\section{Data Entry Procedures}

Lunches from 117 students were documented for each of the 4 days of data collection. Data from $\sim 420$ lunches were gathered, from which 60 lunches were randomly selected and used for the purpose of the study. All items consumed or partially consumed from each lunch were entered into the Nutribase Pro 10.0 (Cybersoft, Inc, Phoenix, AZ, 2010) nutrition software program $(n=364$ items $)$. This software enables the user to create menus, track food intake, and manage individual dietary needs, which makes it very flexible for various research purposes. To the re- searchers' knowledge, it is the only nutrition software package whose nutrient information has been published, allowing nutrient content of individual items to be verified. ${ }^{13}$ This software has the ability to display the complete nutrient data for $>1$ food item at a time. In addition, it includes an option to use the Canadian Nutrient File, which contain $>38,000$ items in the database, including 74 chain restaurant menus (eg, Pizza Hut, McDonald's), and allows the user to input ingredients to generate a new food item. Furthermore, the data from the software can be exported directly to other types of programs, including Excel (which is often used to manage data for research purposes).

Two research assistants (researcher $A$ and researcher B) were involved in the evaluation of the reliability of entering nutrition data into the software. Researcher A randomly selected 30 lunches and researcher $B$ randomly selected 30 different lunches. A sample of 20-40 observations, which in this case were lunches, has been deemed as an appropriate sample for reliability assessments based on the expected range of intraclass correlation coefficients. ${ }^{14}$ The only criterion given to researchers $\mathrm{A}$ and $\mathrm{B}$ for the selection of their 30 lunches was that neither researcher have any previous knowledge of the content of their respective lunches before the data being entered. The researchers entered their data independently by using the same database (2005 Canadian Nutrient File, Health Canada, Canada), as well as a tailored Personal Food Items (PFI) database from the Nutribase Pro software (version 10.0). All items had been previously entered by other researchers for a different project. The PFI option allows its users to create a customized database. The PFI used in the current study contained highrepetition items from the initial entry for the larger project, as well as items absent from the Canadian Nutrient File.

The researchers used the log sheets for each selected lunch, which included the list of items in the lunch, the amount consumed, and any additional details documented during the data collection (eg, the student may have written an estimated portion size on the log sheet, such as the number of carrots). Furthermore, the digital photographs were used to compliment the log sheets by providing a visual aid to estimate portion size and, in many cases, an image of the food label on prepackaged items. The researchers individually "built" their 30 lunches. For singular ingredient items (eg, apple) the researcher needed to locate the item in the database and estimate the portion size (eg, medium-sized apple). For more complex items, such as a ham sandwich, each individual component of the item needed to be entered separately (ie, 2 slices of white bread, $50 \mathrm{~g}$ of ham, 1 tablespoon of mustard, 1 slice of Kraft Single Slice cheese). Many items were prepackaged, and the nutrition labels facilitated the data entry process. However, when prepackaged items located in the database did not exactly match the description on the label, the label information on the item was retained. Items in the database cannot be altered; therefore, this collection method had to be repeated by each researcher during each individual entry.

\section{Intra-rater and Inter-rater Reliability Procedures}

Data on the 30 lunches initially entered by researcher A were independently re-entered by researcher $B$ within 1 week from the initial entry point. Researcher $\mathrm{B}$ then re-entered the data for her 30 original lunches 4 weeks from the day the initial data entry occurred (Figure). This 4-week delay was implemented to control for recall bias. The interval period for a test-retest design depends on the purpose of the study and can range from 1 hour to 1 year. $^{15}$ The 4 -week period used in this study was deemed lengthy enough to avoid memorization and not too lengthy to forget the inner working of the software program. Inter-rater reliability (researcher A original data entry vs researcher B data re-entry) and intra-rater reliability (researcher B original data entry vs researcher B 4-week data re-entry) were assessed by using intraclass correlation coefficients (ICCs) (2-way random model; absolute agreement). ICCs were generated for total kilocalories from all items, total kilocalories from food only, total kilocalories from beverages only, total grams 

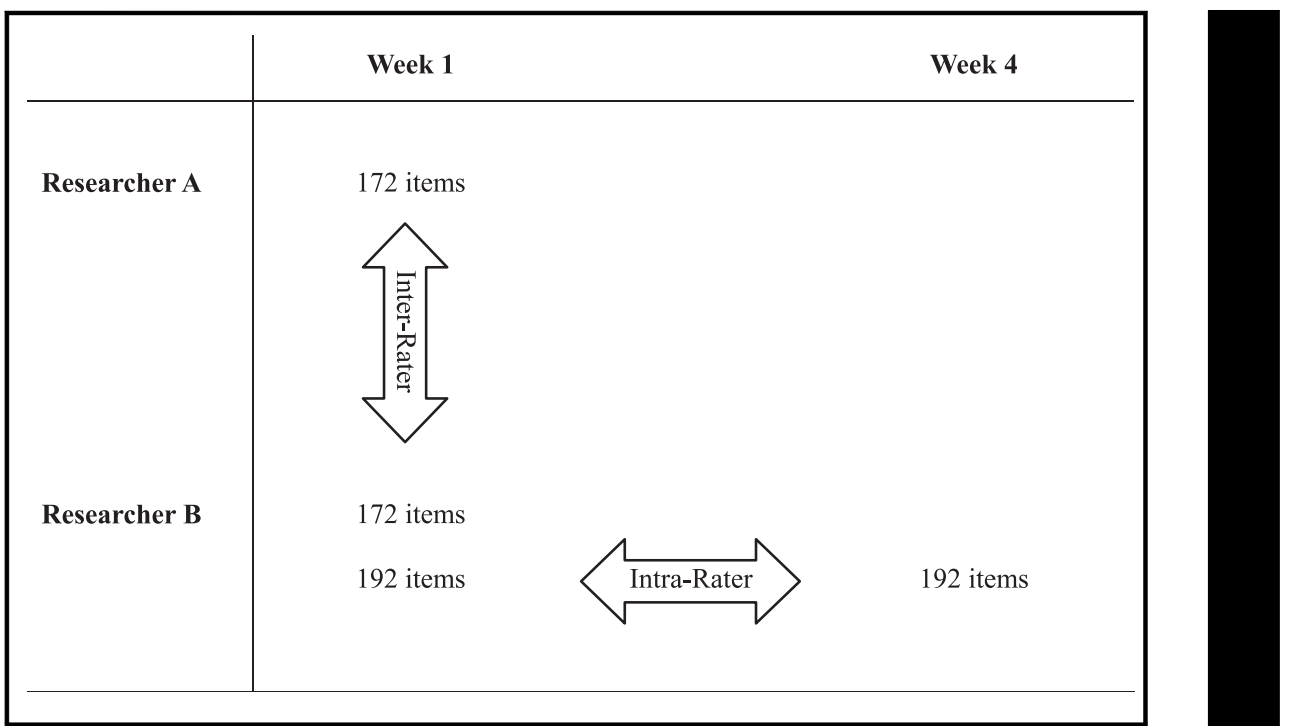

Figure. Inter-rater and intra-rater methodological framework.

from the macronutrients (ie, fat, protein, carbohydrates), as well as sugar specifically, and selected minerals (ie, sodium, calcium) and vitamins (ie, vitamin A, folate, vitamin C) that were considered clinically relevant.

\section{RESULTS}

\section{Sample}

Among the 117 students who participated in the larger study, the mean \pm SD age of the participants was 10.4 \pm 1.26 years; data were collected from 50 boys (42.7\%) and 67 $(57.3 \%)$ girls. Table 1 provides a general description of the home-packed meals for the 60 lunches used in the study. Among the 364 food items used in the study, $54 \%(n=196)$ of the items were prepackaged with a nutrition label.

\section{DISCUSSION}

Streiner and Norman ${ }^{15}$ provide evidence of a range of acceptability for reliability assessments, including a minimum of 0.70 when a scale is used for research purposes and 0.90 for clinical use. The standard of acceptability applied for the current research was 0.80 . The results of the current study, for the most part, confirm both the inter-rater and intrarater reliability of using daily food logs, photographs, and a software application to assess the caloric content of home-packed lunches in a school setting. As expected, the overall intrarater reliability coefficients were superior to the inter-rater reliability coefficients, even after a significant delay in the data re-entry process. In general, the results suggest this method is highly reliable when assessing caloric content, including individual macronutrient values and sugar. However, caution is warranted in using this method to assess certain mineral and vitamin content, particularly among different software users, because 3 of the 5 interuser assessment correlations fell below the standards of acceptability.

To date, most studies evaluating the reliability of assessing food consumption among children have done so in a controlled or cafeteria

Table 1. Description of Home-packed Lunch Contents $(n=60)$

\begin{tabular}{|c|c|c|c|c|}
\hline Lunches & $\begin{array}{l}\text { Total No. } \\
\text { of Items }\end{array}$ & $\begin{array}{l}\text { Total No. of } \\
\text { Food Items }\end{array}$ & $\begin{array}{l}\text { Total No. of } \\
\text { Beverages }\end{array}$ & $\begin{array}{l}\text { Total No. of } \\
\text { Labeled Items }\end{array}$ \\
\hline $\begin{array}{c}\text { Researcher A, } \\
30 \text { lunches }\end{array}$ & 172 & 135 (78.5\%) & 37 (21.5\%) & 88 (51.2\%) \\
\hline $\begin{array}{c}\text { Researcher B, } \\
30 \text { lunches }\end{array}$ & 192 & 150 (78.1\%) & 42 (21.9\%) & 108 (56.3\%) \\
\hline
\end{tabular}

茫

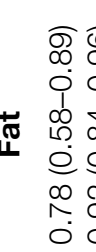

क

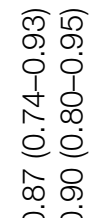

है

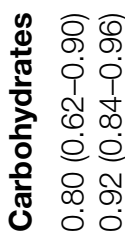

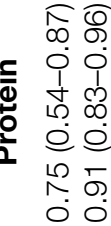

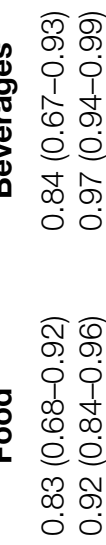

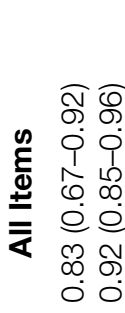


Minerals

\section{Correlation}

Inter-rater

Intra-rater
Sodium

$0.65(0.38-0.81)$

$0.94(0.87-0.97)$
Calcium

$0.83(0.67-0.92)$

$0.98(0.96-0.99)$

Vitamins

Vitamin A

$0.42(0.09-0.68)$

$0.87(0.74-0.94)$
Folate

$0.33(-0.01-0.61)$
Vitamin C

$0.80(0.63-0.90)$

$0.65(0.39-0.82)$

Note: Confidence intervals are given in parentheses.

setting. ${ }^{7-9}$ Cafeterias generally offer a structured menu with controlled portion sizes and a limited variety of items. As such, the number of decisions made by those entering data from home-packed lunches, such as within the current study, is far greater than in a cafeteria setting. The inter-rater ICCs for kilocalories and macronutrients reported in this study (range 0.75-0.87) are strong; however, they are lower than those previously reported (range 0.890.95). ${ }^{7}$ Such differences may be the result of the context (ie, home-packed lunches vs cafeteria-served lunches) of the current study. Notably, some ICCs were below the standard of acceptability; however, they were within close proximity of 0.80 , particularly when considering their CIs (protein 0.54-0.87; fat 0.58-0.89) clearly overlapped the expected ICC (Table 2).

A unique aspect of the current research is that coefficients were also reported for selected micronutrients (Table 3). Doing so revealed that the inter-rater ICC for sodium and intrarater ICC for vitamin $\mathrm{C}$ were moderate. Of greater concern, 2 other ICCs for the selected micronutrients (interrater folate and vitamin A) fell below the standard of acceptability. Therefore, interpretation of data regarding micronutrients (and particularly vitamins) produced using this method must be done so with caution.

Kilocalorie reliability for food and beverages were also analyzed separately, given the general interest in understanding the influence of beverage consumption on child obesity. According to Wang et al, ${ }^{16}$ for nearly all children and adolescents, 10\% of their daily caloric intake comes from fruit juices and sugar-sweetened beverages. Based on these analyses, the inter-rater/intra-rater reliability of beverage data is higher than food alone. This finding is similar to results found by Nicklas et $\mathrm{al}^{10}$ when assessing the accuracy of estimating portion size. These researchers reported greater accuracy in estimated weight (grams) among beverages when isolated from their food data.

Thus, this method of data entry is feasible and its reliability is promising for macronutrient investigations. Continued assessment of this method for investigations related to micronutrient content is recommended.

\section{IMPLICATIONS FOR RESEARCH AND PRACTICE}

The current study offers an inexpensive, noninvasive, reliable method to assess caloric content of homepacked lunches in a school setting. This method seems to be translatable to other meal assessments, such as for assessing packed lunches in the workplace or for seniors in their home. The combination of a log sheet and digital photograph increased the detail and availability of information for those entering the food data. To the researchers' knowledge, this is the first study to assess the reliability of gathering objective food data in an unstructured school setting, and the results are promising.

However, the reader should also consider the following limitations for future applications of this method. First, whether the quantity estimations of food items between researchers were accurately measured cannot be confirmed. The photographs contained an object of known size for comparison; nevertheless, researcher interpretation of serving size and quantity is subjective.

Second, although the Nutribase Pro 10.0 database has published the nutritional content information for all items, which can therefore be individually examined for accuracy, the accuracy of the program's output data relies primarily on its reviews by nutrition professionals who use it and have compared it with similar programs. To date, no scientific study has been performed comparing the accuracy of nutrient information outputs between computer software programs such as Nutribase Pro. Thus, future research should include assessing the concurrent validity of the Nutribase program and other nutrition software programs.

Third, in this study, only 2 researchers entered the data and only 1 intra-rater time interval was used. Additional data points could have been considered. Future research should include additional raters, and all researchers should participate in intra-rater assessment. Doing so may increase confidence in the reliability assessments.

This software is primarily marketed for use as a dietary management tool, and the use of this software is recommended for research purposes. Use of a dietary journal is recommended to complement the digital photograph methods. For instance, the specific content of a sandwich or the number of carrot sticks in a container were captured in the daily $\log$, something that would not have been accurately recorded if photographs alone were used. Conversely, the photograph could also have clarified inaccuracies in the daily log. For future reference, a detailed audit trail of the decisions made during the data entry process (eg, brand of food selected) is recommended. This will be particularly useful if multiple researchers are entering data simultaneously or using the same data set for separate research projects.

\section{ACKNOWLEDGMENT}

This project was financially supported by the Laurentian University Research Fund. 
712 Gauthier et al

\section{REFERENCES}

1. McPherson SR, Hoelscher DM, Alexander M, Scanlon KS, Serdula MK. Dietary assessment methods among school-aged children: validity and reliability. Prev Med. 2000;31:S11-S33.

2. Rockett HR, Berkey CS, Colditz GA. Evaluation of dietary assessment instruments in adolescents. Curr Opin Clin Nutr. 2003;6:557-562.

3. Livingstone MB, Robson PJ, Wallace JM. Issues in dietary intake assessment of children and adolescents. Br J Nutr. 2004;92:S213-S222.

4. Livingstone MB, Robson PJ. Measurement of dietary intake in children. Proc Nutr Soc. 2000;59:279-293.

5. Ngo J, Engelen A, Molag M, Roesle J, Garcia-Segovia P, Serra-Majem L. A review of the use of information and communication technologies for dietary assessment. Br J Nutr. 2009;101: S102-S112.

6. Boushey CJ, Kerr DA, Wright J, Lutes KD, Ebert DS, Delp EJ. Use of

Journal of Nutrition Education and Behavior • Volume 45, Number 6, 2013

technology in children's dietary assessment. Eur J Clin Nutr. 2009;63(suppl 1):S50-S57.

7. Martin CK, Newton RL, Anton SD. Measurement of children's food intake with digital photography and the effects of second servings upon food intake. Eat Behav. 2007;8:148-156.

8. Swanson M. Digital photography as a tool to measure school cafeteria consumption. J School Health. 2008;78: 432-437.

9. Small L, Sidora-Arcoleo K, Vaughan L, Creed-Capsel J, Chung K, Stevens C. Validity and reliability of photographic diet diaries for assessing dietary intake among young children. Infant Child Adolesc Nutr. 2009;1:27-36.

10. Nicklas TA, O'Neil CE, Stuff J, Goodell LS, Liu Y, Martin CK. Validity and feasibility of a digital diet estimation method for use with preschool children: a pilot study. J Nutr Educ Behav. 2012;44:618-623.

11. World Health Organization. WHO Forum and Technical Meeting on
Population-based Prevention Strategies for Childhood Obesity. http://www.who .int/dietphysicalactivity/childhood/ report/en/. Accessed May 9, 2013.

12. World Health Organization. Global Strategy on Diet, Physical Activity and Health-Childhood Overweight and Obesity. http://www.who.int/ dietphysicalactivity/childhood/en/. Accessed May 9, 2013.

13. Ulene A. The Nutribase Nutrition Facts Desk Reference. New York, NY: Penguin Publishing; 2001.

14. Walter SD, Eliasziw M, Donner A. Sample size and optimal designs for reliability studies. Stats Med. 1998;17:101-110.

15. Streiner DL, Norman GR. Health Measurement Scales: A Practical Guide to Their Development and Use. 4th ed. Oxford, UK: Oxford University Press; 2008.

16. Wang YC, Ludwig DS, Sonneville K, Gortmaker SL. Impact of change in sweetened caloric beverage consumption on energy intake among children and adolescents. Arch Pediatr Adolesc Med. 2009;163:336-343. 